PROCEEDINGS OF THE

AMERICAN MATHEMATICAL SOCIETY

Volume 125, Number 4, April 1997, Pages 1111-1121

S 0002-9939(97)03661-7

\title{
A CLASSIFICATION OF TRIGONOMETRICAL THIN SETS AND THEIR INTERRELATIONS
}

\author{
PETER ELIAS̆
}

(Communicated by Andreas R. Blass)

\begin{abstract}
We introduce a uniform way of classifying thin sets of harmonic analysis related to absolute convergence of trigonometric series. This classification covers classical classes $\left(\mathcal{D}, \mathcal{P} \mathcal{D}, \mathcal{A}, \mathcal{N}_{0}, \mathcal{N}\right)$ and yields two new ones $\left(\mathcal{B}_{0}\right.$ and $\mathcal{B}$ ). We study interrelation between these classes concerning combinatorial structure of thin sets.
\end{abstract}

In 1938 J. Marcinkiewicz [8] introduced the notion of N-set (in honour of V. V. Niemytzki): a set $X \subseteq[0,1]$ is an $\mathrm{N}$-set iff there is a trigonometric series

$$
\frac{a_{0}}{2}+\sum_{n=1}^{\infty}\left(a_{n} \cos 2 \pi n x+b_{n} \sin 2 \pi n x\right)
$$

absolutely converging on $X$ with $\sum_{n=1}^{\infty}\left(\left|a_{n}\right|+\left|b_{n}\right|\right)=\infty$ (i.e. not converging absolutely everywhere).

We can equivalently define the N-sets assuming that $a_{n}=0$ for all $n$.

Modifying this definition we obtain a number of types of trigonometrical thin sets, depending on the form of terms in the series and on the convergence used. All but two of the considered classes are equal to the classes of D-, PD-, A-, $\mathrm{N}_{0^{-}}$and $\mathrm{N}$-sets, known from the literature (e.g. [1], [3], [7]). We introduce the classes of $\mathrm{B}_{0^{-}}$ and B-sets, which are new, although already implicitly considered.

It is known that all the classes mentioned above differ from each other (see e.g. [7]). In section 2 we generalize this fact. We get an estimation for the minimum size of a family of sets from one class which covers any set belonging to the other one.

Our notation follows that of [4]. Concerning historical notes we refer again to [4].

The author would like to express his thanks to L. Bukovský, who supervised his master thesis [6] on which this paper is based, and to M. Repický, who helped him with infinite combinatorics.

\section{Classification}

Let $\left\{n_{k}\right\}_{k \in \omega}$ denote an increasing sequence of positive integers and $\left\{\varrho_{k}\right\}_{k \in \omega}$ a sequence of positive reals. We denote by $\|x\|$ the distance of a real $x$ to the nearest

Received by the editors June 8, 1995 and, in revised form, October 11, 1995.

1991 Mathematics Subject Classification. Primary 42A28; Secondary 04A20.

This work was supported by grant 2/1224/94 of Slovak Grant Agency.

(C)1997 American Mathematical Society 
integer. Since $2\|x\| \leq|\sin \pi x| \leq \pi\|x\|$, we can in our consideration replace $|\sin \pi x|$ and $\|x\|$ by each other.

Let us recall the definitions of classical thin sets, related to absolute convergence of trigonometric series:

Definition 1.1. A set $X \subseteq[0,1]$ is

(1) a D-set (a Dirichlet set) iff there exists a sequence $\left\{n_{k}\right\}_{k \in \omega}$ such that $\left\|n_{k} x\right\|$ converges uniformly to 0 on $X$,

(2) a PD-set (a pseudo-Dirichlet set) iff there exists a sequence $\left\{n_{k}\right\}_{k \in \omega}$ such that $\left\|n_{k} x\right\|$ converges quasinormally to 0 on $X$,

(3) an A-set (an Arbault set) iff there exists a sequence $\left\{n_{k}\right\}_{k \in \omega}$ such that $\left\|n_{k} x\right\|$ converges pointwise to 0 on $X$,

(4) an $\mathrm{N}_{0}$-set iff there exists a sequence $\left\{n_{k}\right\}_{k \in \omega}$ such that $\sum_{k=0}^{\infty}\left\|n_{k} x\right\|<\infty$ for $x \in X$,

(5) an N-set iff there exist sequences $\left\{\varrho_{k}\right\}_{k \in \omega}$ and $\left\{n_{k}\right\}_{k \in \omega}$ such that $\sum_{k=0}^{\infty} \varrho_{k}=$ $\infty$ and $\sum_{k=0}^{\infty} \varrho_{k}\left\|n_{k} x\right\|<\infty$ for $x \in X$.

Now define two new classes.

Definition 1.2. A set $X \subseteq[0,1]$ is

(6) a $\mathrm{B}_{0}$-set iff there exist a sequence $\left\{n_{k}\right\}_{k \in \omega}$ and a real constant $c$ such that $\sum_{k=0}^{\infty}\left\|n_{k} x\right\| \leq c$ for $x \in X$

(7) a B-set iff there exist sequences $\left\{\varrho_{k}\right\}_{k \in \omega},\left\{n_{k}\right\}_{k \in \omega}$ and a real constant $c$ such that $\sum_{k=0}^{\infty} \varrho_{k}=\infty$ and $\sum_{k=0}^{\infty} \varrho_{k}\left\|n_{k} x\right\| \leq c$ for $x \in X$.

The classes of all $\mathrm{D}_{-}, \mathrm{PD}-, \mathrm{A}-, \mathrm{N}_{0^{-}}, \mathrm{N}_{-}, \mathrm{B}_{0^{-}}$and $\mathrm{B}$-sets will be denoted by $\mathcal{D}, \mathcal{P} \mathcal{D}, \mathcal{A}, \mathcal{N}_{0}, \mathcal{N}, \mathcal{B}_{0}$ and $\mathcal{B}$, respectively.

We can see that all definitions above have the same form. Only two parameters are changing: the type of convergence and the condition put on the coefficients $\varrho_{k}$.

We will study also some other possibilities for these parameters.

Let us consider the following conditions for the sequence $\left\{\varrho_{k}\right\}_{k \in \omega}$ :

(i) $\sum_{k=0}^{\infty} \varrho_{k}=\infty$,

(ii) $\varrho_{k}$ does not tend to 0 ,

(iii) $\varrho_{k}$ tends to infinity.

Let us consider also the following types of convergence, where $\left\{f_{k}\right\}_{k \in \omega}$ is a sequence of non-negative functions defined on a set $X$ :

(P) $\left\{f_{k}\right\}_{k \in \omega}$ converges pointwise to 0 on $X$;

(QN) $\left\{f_{k}\right\}_{k \in \omega}$ converges quasinormally to 0 on $X$;

(U) $\left\{f_{k}\right\}_{k \in \omega}$ converges uniformly to 0 on $X$;

(PS) $\sum_{k=0}^{\infty} f_{k}(x)$ converges pointwise on $X$, i.e. $\sum_{k=0}^{\infty} f_{k}(x)<\infty$ for $x \in X$;

(QNS) $\sum_{k=0}^{\infty} f_{k}(x)$ converges quasinormally on $X$, i.e. the sequence of its partial sums $\sum_{k=0}^{n} f_{k}(x)$ converges quasinormally on $X$;

(US) $\sum_{k=0}^{\infty} f_{k}(x)$ converges uniformly on $X$, i.e. the sequence of its partial sums $\sum_{k=0}^{n} f_{k}(x)$ converges uniformly on $X$;

(PNS) $\sum_{k=0}^{\infty} f_{k}(x)$ converges pseudonormally on $X$, i.e. there is a sequence of positive reals $\left\{\varepsilon_{k}\right\}_{k \in \omega}$ such that $\sum_{k=0}^{\infty} \varepsilon_{k}<\infty$ and $\forall x \in X \forall^{\infty} k f_{k}(x) \leq \varepsilon_{k}$;

(NS) $\sum_{k=0}^{\infty} f_{k}(x)$ normally converges on $X$, i.e. there is a sequence of positive reals $\left\{\varepsilon_{k}\right\}_{k \in \omega}$ such that $\sum_{k=0}^{\infty} \varepsilon_{k}<\infty$ and $\forall x \in X \forall k f_{k}(x) \leq \varepsilon_{k}$

(BS) $\sum_{k=0}^{\infty} f_{k}(x)$ is bounded on $X$, i.e. there is a real $c$ such that $\sum_{k=0}^{\infty} f_{k}(x) \leq c$ for $x \in X$. 
Let us note that the following implications hold true and that in the general case no other one can be added:

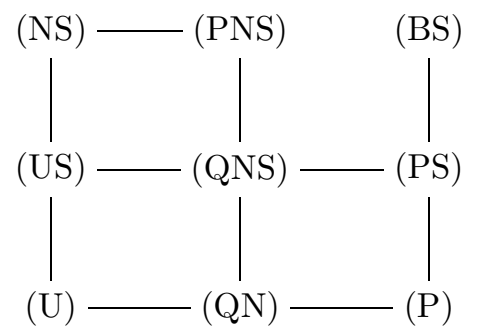

Our aim is to examine all combinations of the properties (i)-(iii), (P)-(BS).

Definition 1.3. Let (a) be one of the conditions (i)-(iii) and (b) one of the convergences $(\mathrm{P})-(\mathrm{BS})$. Denote by $\mathcal{C}_{b}^{a}$ the class of all sets $X \subseteq[0,1]$ for which there exists a sequence of the terms $f_{k}(x)=\varrho_{k}\left\|n_{k} x\right\|$ such that the sequences $\left\{\varrho_{k}\right\}_{k \in \omega}$ and $\left\{f_{k}\right\}_{k \in \omega}$ satisfy the conditions (a) and (b).

It is easy to see that replacing the condition (ii) by $\lim \inf _{k \rightarrow \infty} \varrho_{k}>0$ or $\forall k \in$ $\omega \varrho_{k}=1$ leaves the corresponding classes unchanged. Similarly we may replace the condition (iii) by $\lim \sup _{k \rightarrow \infty} \varrho_{k}=\infty$.

The following simple facts hold true:

Proposition 1.4. Let $\left\{h_{k}\right\}_{k \in \omega}$ denote a sequence of non-negative functions and $\left\{\sigma_{k}\right\}_{k \in \omega}$ a sequence of positive reals.

(1) There exists a sequence $\left\{\varrho_{k}\right\}_{k \in \omega}$ such that $\sum \varrho_{k}=\infty$ and $\lim \varrho_{k}=0$. If $\lim \varrho_{k}=0$ and there is a real $c$ such that $\forall x \in X \forall k \in \omega h_{k}(x) \leq c$, then $\varrho_{k} h_{k}(x)$ converges uniformly to 0 on every set $X$.

(2) If $\sum \varrho_{k}=\infty$ (resp. $\left.\lim \varrho_{k}=\infty\right)$, then there exists a sequence $\left\{\sigma_{k}\right\}_{k \in \omega}$ such that $\lim \sigma_{k}=0$ and $\sum \sigma_{k} \varrho_{k}=\infty\left(\right.$ resp. $\left.\lim \sigma_{k} \varrho_{k}=\infty\right)$.

(3) If $\varrho_{k} h_{k}(x)$ converges pointwise to 0 on $X$ and $\lim \sigma_{k}=0$, then $\sigma_{k} \varrho_{k} h_{k}(x)$ converges quasinormally to 0 on $X$.

(4) If $\sum \varrho_{k} h_{k}(x)$ converges pointwise on $X$ and $\lim \sigma_{k}=0$, then $\sum \sigma_{k} \varrho_{k} h_{k}(x)$ converges quasinormally on $X$.

(5) If $\sum \varrho_{k} h_{k}(x)$ is bounded on $X$ and $\lim \sigma_{k}=0$, then $\sum \sigma_{k} \varrho_{k} h_{k}(x)$ converges uniformly on $X$.

(6) If $\varrho_{k} h_{k}(x)$ converges quasinormally (resp. uniformly) to 0 on $X$, then there exists an increasing sequence $\left\{k_{j}\right\}_{j \in \omega}$ such that $\sum \varrho_{k_{j}} h_{k_{j}}(x)$ converges pseudonormally (resp. normally) on $X$.

(7) If $\sum \varrho_{k}=\infty$ and $\sum \varrho_{k} h_{k}(x)$ converges pseudonormally (resp. normally) on $X$, then there exists an increasing sequence $\left\{k_{j}\right\}_{j \in \omega}$ such that $h_{k_{j}}(x)$ converges quasinormally (resp. uniformly) to 0 on $X$.

(8) If $h_{k}(x)$ converges quasinormally (resp. uniformly) to 0 on $X$, then there exists a sequence $\left\{\varrho_{k}\right\}_{k \in \omega}$ such that $\lim \varrho_{k}=\infty$ and $\varrho_{k} h_{k}(x)$ converges quasinormally (resp. uniformly) to 0 on $X$.

Put $h_{k}(x)=\left\|n_{k} x\right\|$. Then (1) implies that the classes $\mathcal{C}_{\mathrm{P}}^{\mathrm{i}}, \mathcal{C}_{\mathrm{QN}}^{\mathrm{i}}, \mathcal{C}_{\mathrm{U}}^{\mathrm{i}}$ consist of all subsets of $[0,1]$. From $(2)$ and (3) we get $\mathcal{C}_{\mathrm{P}}^{\mathrm{iii}}=\mathcal{C}_{\mathrm{QN}}^{\mathrm{iii}}$, from (2) and (4) $\mathcal{C}_{\mathrm{PS}}^{\mathrm{i}}=$ $\mathcal{C}_{\mathrm{QNS}}^{\mathrm{i}}, \mathcal{C}_{\mathrm{PS}}^{\mathrm{iii}}=\mathcal{C}_{\mathrm{QNS}}^{\mathrm{iii}}$, and from (2) and (5) $\mathcal{C}_{\mathrm{BS}}^{\mathrm{i}}=\mathcal{C}_{\mathrm{US}}^{\mathrm{i}}, \mathcal{C}_{\mathrm{BS}}^{\mathrm{iii}}=\mathcal{C}_{\mathrm{US}}^{\mathrm{iii}}$. From (6) we can conclude that $\mathcal{C}_{\mathrm{QN}}^{\mathrm{ii}}=\mathcal{C}_{\mathrm{QNS}}^{\mathrm{ii}}=\mathcal{C}_{\mathrm{PNS}}^{\mathrm{ii}}, \mathcal{C}_{\mathrm{QN}}^{\mathrm{iii}}=\mathcal{C}_{\mathrm{QNS}}^{\mathrm{iii}}=\mathcal{C}_{\mathrm{PNS}}^{\mathrm{iii}}, \mathcal{C}_{\mathrm{U}}^{\mathrm{ii}}=\mathcal{C}_{\mathrm{US}}^{\mathrm{ii}}=\mathcal{C}_{\mathrm{NS}}^{\mathrm{ii}}$ and 
$\mathcal{C}_{\mathrm{U}}^{\mathrm{iii}}=\mathcal{C}_{\mathrm{US}}^{\mathrm{iii}}=\mathcal{C}_{\mathrm{NS}}^{\mathrm{iii}}$. Assertion (7) implies that $\mathcal{C}_{\mathrm{PNS}}^{\mathrm{i}}=\mathcal{C}_{\mathrm{QN}}^{\mathrm{ii}}, \mathcal{C}_{\mathrm{NS}}^{\mathrm{i}}=\mathcal{C}_{\mathrm{U}}^{\mathrm{ii}}$ and assertion (8) that $\mathcal{C}_{\mathrm{QN}}^{\mathrm{ii}}=\mathcal{C}_{\mathrm{QN}}^{\mathrm{iii}}, \mathcal{C}_{\mathrm{U}}^{\mathrm{ii}}=\mathcal{C}_{\mathrm{U}}^{\mathrm{iii}}$.

Hence every class $\mathcal{C}_{b}^{a}$ is equal to one from Definitions 1.1, 1.2. In the following table all denotes the class of all subsets of $[0,1]$ :

\begin{tabular}{|r|c|c|c|c|c|c|c|c|c|}
\hline & $(\mathrm{P})$ & $(\mathrm{QN})$ & $(\mathrm{U})$ & $(\mathrm{PS})$ & $(\mathrm{QNS})$ & $(\mathrm{US})$ & $(\mathrm{PNS})$ & $(\mathrm{NS})$ & $(\mathrm{BS})$ \\
\hline (i) & all & all & all & $\mathcal{N}$ & $\mathcal{N}$ & $\mathcal{B}$ & $\mathcal{P} \mathcal{D}$ & $\mathcal{D}$ & $\mathcal{B}$ \\
\hline (ii) & $\mathcal{A}$ & $\mathcal{P} \mathcal{D}$ & $\mathcal{D}$ & $\mathcal{N}_{0}$ & $\mathcal{P} \mathcal{D}$ & $\mathcal{D}$ & $\mathcal{P D}$ & $\mathcal{D}$ & $\mathcal{B}_{0}$ \\
\hline (iii) & $\mathcal{P} \mathcal{D}$ & $\mathcal{P D}$ & $\mathcal{D}$ & $\mathcal{P D}$ & $\mathcal{P} \mathcal{D}$ & $\mathcal{D}$ & $\mathcal{P D}$ & $\mathcal{D}$ & $\mathcal{D}$ \\
\hline
\end{tabular}

The inclusions between these classes are the following $(\rightarrow$ means $\subseteq$ ):

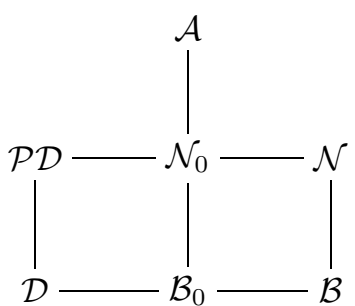

It is known that all these inclusions are proper and that no other one holds.

The following fact was proved by R. Salem [10] for N-sets. However, it holds for other classes too.

Proposition 1.5. In Definition 1.3 we may replace $\left\|n_{k} x\right\|$ by $\left\|\nu_{k} x+\alpha_{k}\right\|$, where $\left\{\nu_{k}\right\}_{k \in \omega}$ is an unbounded sequence of positive reals and $\left\{\alpha_{k}\right\}_{k \in \omega}$ is a sequence of reals.

Proof. Applying Proposition 1.4 to the functions $h_{k}(x)=\left\|\nu_{k} x+\alpha_{k}\right\|$ we obtain an identical table for classes defined using $\left\|\nu_{k} x+\alpha_{k}\right\|$ instead of $\left\|n_{k} x\right\|$. Therefore it is enough to consider the classes from Definitions 1.1 and 1.2. We give the proof only for cases of A- and N-sets; for other classes it goes similarly.

(1) Suppose that $\lim _{k \rightarrow \infty}\left\|\nu_{k}+\alpha_{k}\right\|=0$ for $x \in X$. There exists an increasing sequence $\left\{k_{j}\right\}_{j \in \omega}$ such that $\lim _{j \rightarrow \infty}\left\|\nu_{k_{j+1}}-\nu_{k_{j}}\right\|=0$ and $\lim _{j \rightarrow \infty}\left\|\alpha_{k_{j+1}}-\alpha_{k_{j}}\right\|=0$. Let $n_{j}$ be the nearest integer to $\nu_{k_{j+1}}-\nu_{k_{j}}$. We may suppose that $\left\{n_{j}\right\}_{j \in \omega}$ is an increasing sequence of positive integers. For all $x \in[0,1]$ and $j \in \omega$ we have $\left\|n_{j} x\right\| \leq\left\|\nu_{k_{j+1}} x+\alpha_{k_{j+1}}\right\|+\left\|\nu_{k_{j}} x+\alpha_{k_{j}}\right\|+\left\|\nu_{k_{j+1}}-\nu_{k_{j}}\right\| x+\left\|\alpha_{k_{j+1}}-\alpha_{k_{j}}\right\|$, and therefore $\lim _{j \rightarrow \infty}\left\|n_{j} x\right\|=0$ for $x \in X$.

(2) Assume that $\sum_{k=0}^{\infty} \varrho_{k}=\infty$. There exists a sequence $\left\{s_{k}\right\}_{k \in \omega}$ of positive integers such that $\sum_{k=0}^{\infty} \varrho_{k} s_{k}^{-2}=\infty$, while $\sum_{k=0}^{\infty} \varrho_{k} s_{k}^{-3}<\infty$. For every $k \in \omega$ there is an integer $p_{k}$ such that $1 \leq p_{k} \leq s_{k}^{2},\left\|p_{k} \nu_{k}\right\| \leq s_{k}^{-1}$ and $\left\|p_{k} \alpha_{k}\right\| \leq s_{k}^{-1}$. Let $n_{k}$ be the nearest integer to $p_{k} \nu_{k}$. We have $\left\|n_{k} x\right\| \leq p_{k}\left\|\nu_{k} x+\alpha_{k}\right\|+\left\|p_{k} \nu_{k}\right\| x+$ $\left\|p_{k} \alpha_{k}\right\|$ and hence $\varrho_{k} s_{k}^{-2}\left\|n_{k} x\right\| \leq \varrho_{k}\left\|\nu_{k} x+\alpha_{k}\right\|+\varrho_{k} s_{k}^{-3}(x+1)$. We can see that $\sum_{k=0}^{\infty} \varrho_{k} s_{k}^{-2}\left\|n_{k} x\right\|<\infty$, whenever $\sum_{k=0}^{\infty} \varrho_{k}\left\|\nu_{k} x+\alpha_{k}\right\|<\infty$.

\section{INTERRELATIONS BETWEEN THE CLASSES}

Notice that all non-inclusions in the diagram (D) are based on the following four: $\mathcal{B}_{0} \nsubseteq \subseteq \mathcal{P D}, \mathcal{P D} \nsubseteq \mathcal{B}, \mathcal{B} \nsubseteq \mathcal{A}$ and $\mathcal{A} \nsubseteq \mathcal{N}$. Non-inclusion $\mathcal{P} \mathcal{D} \nsubseteq \mathcal{B}$ is easy because B-sets are always nowhere dense, while there are PD-sets dense in [0,1] (e.g. every countable set is a PD-set). In [7] S. Kahane gave examples of an $\mathrm{N}_{0^{-}}$-set (actually 
$\mathrm{B}_{0}$-set) which is not a countable union of PD-sets, an N-set (actually B-set) which is not a countable union of A-sets, and an A-set which is not a countable union of $\mathrm{N}$-sets. Using the idea contained in his constructions we prove more informative facts about interrelations between these classes.

Let us start with some simple observations.

Lemma 2.1. Let $x \in[0,1], n$ be a positive integer and $\varepsilon$ be a real, $0 \leq \varepsilon \leq \frac{1}{2}$. Then the following conditions are equivalent:

(1) $|\sin \pi n x| \leq \sin \pi \varepsilon$,

(2) $\|n x\| \leq \varepsilon$,

(3) $x \in \bigcup_{i=0}^{n-1}\left[\frac{i}{n}, \frac{i}{n}+\frac{\varepsilon}{n}\right] \cup\left[\frac{i+1}{n}-\frac{\varepsilon}{n}, \frac{i+1}{n}\right]$.

By an interval we mean a closed, non-empty subinterval of $[0,1]$. We denote by $|I|$ the length of an interval $I$.

Corollary 2.2. Let $I$ be an interval, $n$ a positive integer, and $\varepsilon$ a real, $0 \leq \varepsilon \leq \frac{1}{2}$.

(1) If $|I| \geq \frac{1}{n}$, then there exists an interval $J \subseteq I$ such that $\forall x \in J\|n x\| \leq \varepsilon$ and $|J| \geq \frac{\varepsilon}{n}$

(2) If $|I| \geq \frac{2 \varepsilon}{n}$, then there exists an interval $J \subseteq I$ such that $\forall x \in J\|n x\| \geq \varepsilon$ and $|J| \geq \min \left\{\frac{|I|}{2}-\frac{\varepsilon}{n}, \frac{1-2 \varepsilon}{n}\right\}$.

Lemma 2.3. Let $n, n^{\prime} \geq 2 n$ be positive integers and let $I$ be an interval with $|I| \geq$ $\frac{1}{n}$. Then there exists an interval $J \subseteq I$ such that $\forall x \in J\|n x\| \leq \frac{n}{n^{\prime}}$ and $|J| \geq \frac{1}{n^{\prime}}$.

Proof. Take $\varepsilon=\frac{n}{n^{\prime}} \leq \frac{1}{2}$ in Corollary 2.2.

Let us note that T. Viola in his paper [12] using a similar fact proved that if $\sum_{k=0}^{\infty} \frac{n_{k}}{n_{k+1}}<\infty$, then the set of all $x$, such that the series $\sum_{k=0}^{\infty} \cos 2 \pi n_{k}\left(x+\alpha_{k}\right)$ absolutely converges, is of the cardinality continuum.

Lemma 2.4. Let $n, n^{\prime}, m$ be positive integers and let $\varepsilon$ be a real such that $0<\varepsilon \leq$ $\frac{1}{16}, n \leq 2 m<n^{\prime}$ and $n \leq \varepsilon n^{\prime}$. Let $I$ be an interval with $|I| \geq \frac{1}{n}$. Then there exist intervals $J_{0}, J_{1} \subseteq I$ with disjoint interiors, such that $\forall x \in J_{0} \cup J_{1}\|n x\| \leq$ $8 \varepsilon \wedge\|m x\| \geq \frac{\varepsilon}{2}$ and $\left|J_{0}\right|,\left|J_{1}\right| \geq \frac{1}{n^{\prime}}$.

Proof. By Corollary 2.2 (1) used for $I, n$ and $8 \varepsilon$ there is an interval $I^{\prime} \subseteq I$ such that $\forall x \in I^{\prime}\|n x\| \leq 8 \varepsilon$ and $\left|I^{\prime}\right| \geq \frac{8 \varepsilon}{n}$. Let $I_{0}^{\prime}$ and $I_{1}^{\prime}$ be the left and the right half of $I^{\prime}$. By Corollary 2.2 (2) used for $I_{0}^{\prime}, m$ and $\frac{\varepsilon}{2}$ there is $J_{0} \subseteq I_{0}^{\prime}$ such that $\forall x \in J_{0}\|m x\| \geq \frac{\varepsilon}{2}$ and

$$
\left|J_{0}\right| \geq \min \left\{\frac{2 \varepsilon}{n}-\frac{\varepsilon}{2 m}, \frac{1-\varepsilon}{m}\right\} \geq \min \left\{\frac{2 \varepsilon}{n}-\frac{\varepsilon}{n}, \frac{1}{2 m}\right\} \geq \min \left\{\frac{\varepsilon}{n}, \frac{1}{n^{\prime}}\right\} \geq \frac{1}{n^{\prime}} .
$$

Similarly there is $J_{1} \subseteq I_{1}^{\prime}$ such that $\forall x \in J_{1}\|m x\| \geq \frac{\varepsilon}{2}$ and $\left|J_{1}\right| \geq \frac{1}{n^{\prime}}$.

We will need the following characterizations of two cardinal invariants of the ideal $\mathbb{K}$ of meager sets, due to J. Truss, A. W. Miller [9] and T. Bartoszyński [2]:

$$
\begin{gathered}
\operatorname{add}(\mathbb{K})=\min \{\mathfrak{b}, \operatorname{cov}(\mathbb{K})\} \\
\operatorname{cov}(\mathbb{K})=\min \left\{|X|: X \subseteq \omega_{\omega} \wedge \forall x \in \omega_{\omega} \exists y \in X \forall \forall^{\infty} n \in \omega y(n) \neq x(n)\right\} .
\end{gathered}
$$

Let us recall that

$$
\mathfrak{b}=\min \left\{|X|: X \subseteq \omega_{\omega} \wedge \forall x \in \omega_{\omega} \exists y \in X \exists^{\infty} n \in \omega y(n)>x(n)\right\} .
$$

For basic information see e.g. [5], [11]. 
Theorem 2.5. Assume that $\sum_{k=0}^{\infty} \frac{n_{k}}{n_{k+1}}<\infty$. Let $X$ be the $N_{0}$-set $\{x \in[0,1]$ : $\left.\sum_{k=0}^{\infty}\left\|n_{k} x\right\|<\infty\right\}$, let $\mathcal{Y}$ be a family of $P D$-sets of size $|\mathcal{Y}|<\operatorname{add}(\mathbb{K})$, and let $I_{0}$ be an interval with non-empty interior. Then the set $(X \backslash \cup \mathcal{Y}) \cap I_{0}$ has a perfect subset.

Proof. Omitting finitely many terms from the sequence $\left\{n_{k}\right\}_{k \in \omega}$ we can ensure that $\left|I_{0}\right| \geq \frac{1}{n_{0}}$ and $n_{k+1} \geq 2 n_{k}$ for all $k \in \omega$. Fix a sequence $\left\{\varepsilon_{j}\right\}_{j \in \omega}$ such that $0 \leq \varepsilon_{j} \leq \frac{1}{16}$ for all $j \in \omega$ and $\sum_{j=0}^{\infty} \varepsilon_{j}<\infty$.

Suppose that $\mathcal{Y}=\left\{Y_{\xi}: \xi<\kappa\right\}, \kappa<\operatorname{add}(\mathbb{K})$. For $\xi<\kappa$ let $\left\{m_{l}^{\xi}\right\}_{l \in \omega}$ be an increasing sequence of positive integers and $\left\{\theta_{l}^{\xi}\right\}_{l \in \omega}$ a sequence of positive reals such that $\lim _{l \rightarrow \infty} \theta_{l}^{\xi}=0$ and $\forall x \in Y_{\xi} \forall^{\infty} l\left\|m_{l}^{\xi} x\right\| \leq \theta_{l}^{\xi}$. For $m \in \omega$ let $k(m)$ be such that $n_{k(m)} \leq 2 m<n_{k(m)+1}$. For any $i, j \in \omega$ and $\xi<\kappa$ find an integer $l_{\xi}(i, j)$ such that $k\left(m_{l_{\xi}(i, j)}^{\xi}\right) \geq i$ and $\theta_{l_{\xi}(i, j)}^{\xi}<\frac{\varepsilon_{j}}{2}$. Put $p_{\xi}(i, j)=k\left(m_{l_{\xi}(i, j)}^{\xi}\right)+1$.

Since $\kappa<\mathfrak{b}$, there exists $p \in{ }^{\omega \times \omega} \omega$ such that $\forall \xi<\kappa \forall^{\infty}(i, j) p(i, j) \geq p_{\xi}(i, j)$, i.e. for every $\xi<\kappa$ there is $j_{\xi} \in \omega$ such that if $\min \{i, j\} \geq j_{\xi}$, then $p(i, j) \geq p_{\xi}(i, j)$. Put $p_{0}=0, p_{j+1}=p\left(p_{j}, j\right)$ for $j \in \omega$. Clearly if $j \geq j_{\xi}$, then $p_{j} \leq k\left(m_{l_{\xi}\left(p_{j}, j\right)}\right)<$ $p_{\xi}\left(p_{j}, j\right) \leq p\left(p_{j}, j\right)=p_{j+1}$.

Since $\kappa<\operatorname{cov}(\mathbb{K})$, there exists a sequence $\left\{m_{j}\right\}_{j \in \omega}$ such that $\forall \xi<\kappa \exists^{\infty} j m_{j}=$ $m_{l_{\xi}\left(p_{j}, j\right)}^{\xi}$. Denote $M_{\xi}=\left\{j \in \omega: j \geq j_{\xi} \wedge m_{j}=m_{l_{\xi}\left(p_{j}, j\right)}^{\xi}\right\}, M=\bigcup_{\xi<\kappa} M_{\xi}$ and $N=\left\{k\left(m_{j}\right): j \in M\right\}$. The sets $M_{\xi}, M, N$ are infinite and $m$ is increasing on $M$.

Now let us construct our perfect set by an induction on $k$. Put $\mathcal{T}_{0}=\left\{I_{0}\right\}$. Suppose that $\mathcal{I}_{k}$ is a family of intervals with pairwise disjoint interiors and that $|I| \geq \frac{1}{n_{k}}$ for every $I \in \mathcal{T}_{k}$.

If $k \in \omega \backslash N$, then $n_{k+1} \geq 2 n_{k}$ and by Lemma 2.3 for any $I \in \mathcal{T}_{k}$ there exists $J^{I} \subseteq I$ such that $\forall x \in J^{I}\left\|n_{k} x\right\| \leq \frac{n_{k}}{n_{k+1}}$ and $\left|J^{I}\right| \geq \frac{1}{n_{k+1}}$. Put $\mathcal{T}_{k+1}=\left\{J^{I}: I \in\right.$ $\left.\mathcal{T}_{k}\right\}$.

If $k \in N$, then $k=k\left(m_{j}\right)$ for some $j \in M, n_{k} \leq 2 m_{j}<n_{k+1}$ and $n_{k} \leq \varepsilon_{j} n_{k+1}$. By Lemma 2.4 for any $I \in \mathcal{T}_{k}$ there exist $J_{0}^{I}, J_{1}^{I} \subseteq I$ such that $\forall x \in J_{0}^{I} \cup J_{1}^{I}\left\|n_{k} x\right\| \leq$ $8 \varepsilon_{j} \wedge\left\|m_{j} x\right\| \geq \frac{\varepsilon_{j}}{2}$ and $\left|J_{0}^{I}\right|,\left|J_{1}^{I}\right| \geq \frac{1}{n_{k+1}}$. Put $\mathcal{T}_{k+1}=\left\{J_{0}^{I}, J_{1}^{I}: I \in \mathcal{T}_{k}\right\}$.

The set $P=\bigcap_{k \in \omega} \cup \mathcal{T}_{k}$ is a perfect subset of $I_{0}$. If $x \in P$, then $\sum_{k=0}^{\infty}\left\|n_{k} x\right\|=$ $\sum_{k \in \omega \backslash N}\left\|n_{k} x\right\|+\sum_{j \in M}\left\|n_{k\left(m_{j}\right)} x\right\| \leq \sum_{k \in \omega \backslash N} \frac{n_{k}}{n_{k+1}}+\sum_{j \in M} 8 \varepsilon_{j}<\infty$, and hence $x \in X$. Moreover, for any $\xi<\kappa$ and $j \in M_{\xi}$ we have $m_{j}=m_{l_{\xi}\left(p_{j}, j\right)}^{\xi},\left\|m_{j} x\right\| \geq \frac{\varepsilon_{j}}{2}>$ $\theta_{l_{\xi}\left(p_{j}, j\right)}^{\xi}$, and therefore $x \notin Y_{\xi}$. Hence $P \subseteq X \backslash \cup \mathcal{Y}$.

Theorem 2.6. Assume that $\sum_{k=0}^{\infty} \frac{n_{k}}{n_{k+1}}<\infty$. Let $X$ be the $B_{0}$-set $\{x \in[0,1]$ : $\left.\sum_{k=0}^{\infty}\left\|n_{k} x\right\| \leq c\right\}$, where $c$ is a sufficiently big real, and let $\mathcal{Y}$ be a family of PD-sets of size $|\mathcal{Y}|<\operatorname{add}(\mathbb{K})$. Then the set $X \backslash \bigcup \mathcal{Y}$ has a perfect subset.

Proof. Find $k_{0}$ such that $n_{k+1} \geq 2 n_{k}$ for $k \geq k_{0}$ and take $c>\frac{k_{0}}{2}+\sum_{k=k_{0}}^{\infty} \frac{n_{k}}{n_{k+1}}$. Put $I_{0}=[0,1]$ and choose $\left\{\varepsilon_{j}\right\}_{j \in \omega}$ such that $\frac{k_{0}}{2}+\sum_{k=k_{0}}^{\infty} \frac{n_{k}}{n_{k+1}}+8 \sum_{j=0}^{\infty} \varepsilon_{j} \leq c$. Similarly as in Theorem 2.5 there is a perfect set $P \subseteq I_{0}$ disjoint with every $Y \in \mathcal{Y}$, such that if $x \in P$, then $\sum_{k=k_{0}}^{\infty}\left\|n_{k} x\right\| \leq \sum_{k=k_{0}}^{\infty} \frac{n_{k}}{n_{k+1}}+8 \sum_{j=0}^{\infty} \varepsilon_{j}$, and hence $P \subseteq X$.

Theorem 2.7. Assume that $\lim _{k \rightarrow \infty} \varrho_{k}=0, \sum_{k=0}^{\infty} \varrho_{k}=\infty$ and $\sum_{k=0}^{\infty} \varrho_{k} \frac{n_{k}}{n_{k+1}}<$ $\infty$. Let $X$ be the $N$-set $\left\{x \in[0,1]: \sum_{k=0}^{\infty} \varrho_{k}\left\|n_{k} x\right\|<\infty\right\}$, let $\mathcal{Y}$ be a family of $A$-sets of size $|\mathcal{Y}|<\operatorname{add}(\mathbb{K})$ and let $I_{0}$ be an interval with non-empty interior. Then the set $(X \backslash \bigcup \mathcal{Y}) \cap I_{0}$ has a perfect subset. 
Proof. Fix a real $\varepsilon, 0<\varepsilon \leq \frac{1}{16}$, and a sequence $\left\{\theta_{j}\right\}_{j \in \omega}$ of positive reals such that $\sum_{j=0}^{\infty} \theta_{j}<\infty$. Denote $S=\left\{k \in \omega:\left|I_{0}\right| \geq \frac{1}{n_{k}} \wedge n_{k} \leq \varepsilon n_{k+1}\right\}$. Clearly $\sum_{k \in \omega \backslash S} \varrho_{k}<\infty$, and hence $S$ is infinite. Denote by $S(k)$ the $k$-th member of $S$.

Suppose that $\mathcal{Y}=\left\{Y_{\xi}: \xi<\kappa\right\}, \kappa<\operatorname{add}(\mathbb{K})$. For $\xi<\kappa$ let $\left\{m_{l}^{\xi}\right\}_{l \in \omega}$ be an increasing sequence of positive integers such that $\forall x \in Y_{\xi} \lim _{l \rightarrow \infty}\left\|m_{l}^{\xi} x\right\|=0$. For $m \in \omega$ let $k(m)$ be such that $n_{S(k(m))} \leq 2 m<n_{S(k(m)+1)}$. For any $i, j \in \omega$ and $\xi<\kappa$ find an integer $l_{\xi}(i, j)$ such that $k\left(m_{l_{\xi}(i, j)}^{\xi}\right) \geq i$ and $\varrho_{S\left(k\left(m_{l_{\xi}(i, j)}^{\xi}\right)\right)} \leq \theta_{j}$.

Similarly as in Theorem 2.5 we can find an increasing sequence $\left\{p_{j}\right\}_{j \in \omega}$, a sequence $\left\{m_{j}\right\}_{j \in \omega}$ and for any $\xi<\kappa$ an infinite set $M_{\xi} \subseteq \omega$, such that if $\xi<\kappa$ and $j \in M_{\xi}$, then $m_{j}=m_{l_{\xi}\left(p_{j}, j\right)}^{\xi}, p_{j} \leq k\left(m_{j}\right)<p_{j+1}$ and $\varrho_{S\left(k\left(m_{j}\right)\right)} \leq \theta_{j}$. Denote $M=\bigcup_{\xi<\kappa} M_{\xi}$ and $N=\left\{k\left(m_{j}\right): j \in M\right\}$. Clearly $m$ is increasing on $M$ and $M, N$ are infinite.

We know that $\left|I_{0}\right| \geq \frac{1}{n_{S(0)}}$ and $n_{S(k)} \leq \varepsilon n_{S(k)+1}$ for all $k \in \omega$. By a similar construction as in Theorem 2.5 we can construct a perfect set $P \subseteq I_{0}$ such that if $x \in P$, then $\left\|n_{S(k)} x\right\| \leq \frac{n_{S(k)}}{n_{S(k)+1}}$ for $k \in \omega \backslash N,\left\|n_{S(k)} x\right\| \leq 8 \varepsilon \wedge\left\|m_{j} x\right\| \geq \frac{\varepsilon}{2}$ for $k=k\left(m_{j}\right), j \in M$.

For $x \in P, \sum_{k=0}^{\infty} \varrho_{k}\left\|n_{k} x\right\| \leq \frac{1}{2} \sum_{k \in \omega \backslash S} \varrho_{k}+\sum_{k \in S} \varrho_{k}\left\|n_{k} x\right\|<\infty$ holds, since

$$
\begin{aligned}
\sum_{k \in S} \varrho_{k}\left\|n_{k} x\right\| & =\sum_{k \in \omega \backslash N} \varrho_{S(k)}\left\|n_{S(k)} x\right\|+\sum_{j \in M} \varrho_{S\left(k\left(m_{j}\right)\right)}\left\|n_{S\left(k\left(m_{j}\right)\right)} x\right\| \\
& \leq \sum_{k \in \omega \backslash N} \varrho_{S(k)} \frac{n_{S(k)}}{n_{S(k)+1}}+\sum_{j \in M} 8 \varepsilon \theta_{j} \leq \sum_{k \in S} \varrho_{k} \frac{n_{k}}{n_{k+1}}+8 \varepsilon \sum_{j=0}^{\infty} \theta_{j},
\end{aligned}
$$

and therefore $x \in X$. Moreover, for $\xi<\kappa$ and $j \in M_{\xi}$ we have $\left\|m_{l_{\xi}\left(p_{j}, j\right)}^{\xi} x\right\|=$ $\left\|m_{j} x\right\| \geq \frac{\varepsilon}{2}$, and hence $x \notin Y_{\xi}$. We get $P \subseteq X \backslash \bigcup \mathcal{Y}$.

Theorem 2.8. Assume the hypotheses of Theorem 2.7. Let $X$ be the B-set $\{x \in$ $\left.[0,1]: \sum_{k=0}^{\infty} \varrho_{k}\left\|n_{k} x\right\| \leq c\right\}$, where $c$ is a sufficiently big real, and let $\mathcal{Y}$ be a family of $A$-sets of size $|\mathcal{Y}|<\operatorname{add}(\mathbb{K})$. Then the set $X \backslash \cup \mathcal{Y}$ has a perfect subset.

Proof. Fix a real $\varepsilon, 0<\varepsilon \leq \frac{1}{16}$, and denote $S=\left\{k \in \omega: n_{k} \leq \varepsilon n_{k+1}\right\}$. It is sufficient to take $c>\frac{1}{2} \sum_{k \in \omega \backslash S} \varrho_{k}+\sum_{k \in S} \varrho_{k} \frac{n_{k}}{n_{k+1}}$. Put $I_{0}=[0,1]$ and choose $\left\{\theta_{j}\right\}_{j \in \omega}$ such that $\frac{1}{2} \sum_{k \in \omega \backslash S} \varrho_{k}+\sum_{k \in S} \varrho_{k} \frac{n_{k}}{n_{k+1}}+8 \varepsilon \sum_{j=0}^{\infty} \theta_{j} \leq c$. Similarly as in Theorem 2.7 there exists a perfect set $P \subseteq I_{0}$, disjoint with every $Y \in \mathcal{Y}$, such that if $x \in P$, then $\sum_{k=0}^{\infty} \varrho_{k}\left\|n_{k} x\right\| \leq \frac{1}{2} \sum_{k \in \omega \backslash S} \varrho_{k}+\sum_{k \in S} \varrho_{k} \frac{n_{k}}{n_{k+1}}+8 \varepsilon \sum_{j=0}^{\infty} \theta_{j} \leq c$.

The following lemma appears in [7] in a slightly different form.

Lemma 2.9. Let $\left\{\varrho_{n}\right\}_{n \in \omega}$ be a sequence of positive reals, let $a, b, \theta$ be positive reals and let $I$ be an interval such that $a<b, \theta \leq \frac{1}{14}$ and $|I| \geq \frac{6 \theta}{a}$. Then there exists an interval $J \subseteq I$ such that $\forall x \in J \quad \sum_{a \leq n<b} \varrho_{n}\|n x\| \geq \frac{\theta}{4} \sum_{a \leq n<b} \varrho_{n}$ and $|J| \geq \frac{2 \theta}{b}$.

Proof. Suppose for now that $b \leq 3 a$. There exists $\bar{x}$ such that $\left[\bar{x}-\frac{\theta}{b}, \bar{x}+\frac{3 \theta}{a}+\frac{2 \theta}{b}\right] \subseteq I$. Denote $A=\{n \in \omega: a \leq n<b \wedge\|n \bar{x}\| \geq 2 \theta\}, B=\{n \in \omega: a \leq n<b \wedge\|n \bar{x}\|<2 \theta\}$.

If $\sum_{n \in A} \varrho_{n} \geq \frac{1}{2} \sum_{n \in A \cup B} \varrho_{n}$, put $J=\left[\bar{x}-\frac{\theta}{b}, \bar{x}+\frac{\theta}{b}\right]$. Then for $x \in J$ and $n \in A$ we have $\|n x\| \geq\|n \bar{x}\|-\|n(x-\bar{x})\| \geq 2 \theta-n|x-\bar{x}| \geq 2 \theta-\theta=\theta$. 
Otherwise, if $\sum_{n \in B} \varrho_{n}>\frac{1}{2} \sum_{n \in A \cup B} \varrho_{n}$, put $J=\left[\bar{x}+\frac{3 \theta}{a}, \bar{x}+\frac{3 \theta}{a}+\frac{2 \theta}{b}\right]$. Then for $x \in J$ and $n \in B$ we have $\frac{3 \theta}{a} \leq|x-\bar{x}| \leq \frac{3 \theta}{a}+\frac{2 \theta}{b} \leq \frac{11 \theta}{b}, 3 \theta \leq n|x-\bar{x}| \leq 11 \theta \leq 1-3 \theta$, and hence $\|n x\| \geq\|n(x-\bar{x})\|-\|n \bar{x}\| \geq 3 \theta-2 \theta=\theta$.

In both cases we get $\sum_{a \leq n<b} \varrho_{n} \geq \frac{\theta}{2} \sum_{a \leq n<b} \varrho_{n}$.

In the case that $b>3 a$ put $a_{0}=a, a_{1}=3 a_{0}, a_{2}=3 a_{1}, \ldots, a_{k}=b \leq 3 a_{k-1}$. Denote $A=\left\{n \in \omega: a_{i} \leq n<a_{i+1}\right.$ for $i$ even $\}, B=\left\{n \in \omega: a_{i} \leq n<a_{i+1}\right.$ for $i$ odd $\}$.

If $\sum_{n \in A} \varrho_{n} \geq \frac{1}{2} \sum_{n \in A \cup B} \varrho_{n}$, then iterating the previous consideration we can find intervals $I \supseteq I_{0} \supseteq I_{2} \supseteq \cdots$ such that for $i$ even the following holds:

$$
\left|I_{i}\right| \geq \frac{2 \theta}{a_{i+1}}=\frac{6 \theta}{a_{i+2}} \quad \text { and } \forall x \in I_{i} \quad \sum_{a_{i} \leq n<a_{i+1}} \varrho_{n}\|n x\| \geq \frac{\theta}{2} \sum_{a_{i} \leq n<a_{i+1}} \varrho_{n} .
$$

Similarly, if $\sum_{n \in B} \varrho_{n}>\frac{1}{2} \sum_{n \in A \cup B} \varrho_{n}$, then there exist intervals $I \supseteq I_{1} \supseteq I_{3} \supseteq \ldots$ such that $(*)$ holds for $i$ odd. In both cases let $J$ be the last interval $\left(I_{k-2}\right.$ or $\left.I_{k-1}\right)$. We get $|J| \geq \frac{2 \theta}{b}$ and $\forall x \in J \quad \sum_{a \leq n<b} \varrho_{n}\|n x\| \geq \frac{\theta}{4} \sum_{a \leq n<b} \varrho_{n}$.

Theorem 2.10. Assume that $\lim _{k \rightarrow \infty} \frac{n_{k}}{n_{k+1}}=0$. Let $X$ be the A-set $\{x \in[0,1]$ : $\left.\lim _{k \rightarrow \infty}\left\|n_{k} x\right\|=0\right\}$, let $\mathcal{Y}$ be a family of $N$-sets of size $|\mathcal{Y}|<\operatorname{add}(\mathbb{K})$ and let $I_{0}$ be an interval with non-empty interior. Then the set $(X \backslash \bigcup \mathcal{Y}) \cap I_{0}$ has a perfect subset.

Proof. Omitting finitely many terms from the sequence $\left\{n_{k}\right\}_{k \in \omega}$ we can ensure that $\left|I_{0}\right| \geq \frac{1}{n_{0}}$ and $n_{k+1} \geq 2 n_{k}$ for all $k \in \omega$. Fix a sequence $\left\{\varepsilon_{j}\right\}_{j \in \omega}$ such that $0<\varepsilon_{j} \leq \frac{1}{2}$ and $\lim _{j \rightarrow \infty} \varepsilon_{j}=0$.

Suppose that $\mathcal{Y}=\left\{Y_{\xi}: \xi<\kappa\right\}, \kappa<\operatorname{add}(\mathbb{K})$. For $\xi<\kappa$ let $\left\{\varrho_{n}^{\xi}\right\}_{n \in \omega}$ be a sequence of positive rationals such that $\sum_{n=0}^{\infty} \varrho_{n}^{\xi}=\infty$ and $\forall x \in Y_{\xi} \sum_{n=0}^{\infty} \varrho_{n}^{\xi}\|n x\|<\infty$. Denote $c=\frac{1}{14}$ and fix $\xi<\kappa$. There are two possibilities.

(a) There exists $d>c$ such that

$$
\sum_{k=0}^{\infty} \sum_{c n_{k} \leq n<d n_{k}} \varrho_{n}^{\xi}=\infty
$$

Then we can choose $\theta_{k}^{\xi}$ such that $\theta_{\kappa}^{\xi} \geq d \frac{n_{k}}{n_{k+1}}, \lim _{k \rightarrow \infty} \theta_{k}^{\xi}=0$ and

$$
\sum_{k=0}^{\infty} \theta_{k}^{\xi} \sum_{c n_{k} \leq n<d n_{k}} \varrho_{n}^{\xi}=\infty
$$

Denote $a_{k}^{\xi}=c n_{k}, b_{k}^{\xi}=d n_{k}$ for all $k \in \omega$.

(b) For all $d>c$,

$$
\sum_{k=0}^{\infty} \sum_{c n_{k} \leq n<d n_{k}} \varrho_{n}^{\xi}<\infty
$$

holds. Then for all $d>c$ we have

$$
\sum_{k=0}^{\infty} \sum_{d n_{k} \leq n<c n_{k+1}} \varrho_{n}^{\xi}=\infty
$$


and hence we can choose $d_{k}$ such that $c<d_{k}<c \frac{n_{k+1}}{n_{k}}, \lim _{k \rightarrow \infty} d_{k}=\infty$ and

$$
\sum_{k=0}^{\infty} \sum_{d_{k} n_{k} \leq n<c n_{k+1}} \varrho_{n}^{\xi}=\infty
$$

Denote $a_{k}^{\xi}=d_{k} n_{k}, b_{k}^{\xi}=c n_{k+1}$ and $\theta_{k}^{\xi}=c$ for all $k \in \omega$.

In both cases we have found sequences $\left\{a_{k}^{\xi}\right\}_{k \in \omega},\left\{b_{k}^{\xi}\right\}_{k \in \omega}$ and $\left\{\theta_{k}^{\xi}\right\}_{k \in \omega}$ such that $\sum_{k=0}^{\infty} \theta_{k}^{\xi} \sum_{a_{k}^{\xi} \leq n<b_{k}^{\xi}} \varrho_{n}^{\xi}=\infty$. Moreover, for every $j \in \omega$ there exists $k_{j}^{\xi} \in \omega$ such that for all $k \geq k_{j}^{\xi}$,

$$
\frac{\varepsilon_{j}}{n_{k}} \geq \frac{6 \theta_{k}^{\xi}}{a_{k}^{\xi}}, \quad \frac{2 \theta_{k}^{\xi}}{b_{k}^{\xi}} \geq \frac{2}{n_{k+1}}, \quad \theta_{k}^{\xi} \leq \frac{1}{14} \quad \text { and } \quad a_{k}^{\xi}<b_{k}^{\xi} \leq a_{k+1}^{\xi}
$$

hold. Let us note that we can choose $a_{k}^{\xi}, b_{k}^{\xi}$ and $\theta_{k}^{\xi}$ rational.

For any $i, j \in \omega$ and $\xi<\kappa$ find integers $r_{\xi}(i, j), s_{\xi}(i, j)$ and $p_{\xi}(i, j)$ such that

$$
\bigcup_{r_{\xi}(i, j) \leq k<s_{\xi}(i, j)}^{k_{j}^{\xi} \leq r_{\xi}(i, j)<s_{\xi}(i, j), \quad\left[r_{\xi}(i, j), s_{\xi}(i, j)\right) \subseteq\left[i, p_{\xi}(i, j)\right),}
$$

Since $\kappa<\mathfrak{b}$, there exists $p \in \omega \times \omega_{\omega}$ such that $\forall \xi<\kappa \forall^{\infty}(i, j) p(i, j) \geq p_{\xi}(i, j)$, i.e. for every $\xi<\kappa$ there is $j_{\xi} \in \omega$ such that if $\min \{i, j\} \geq j_{\xi}$, then $p(i, j) \geq p_{\xi}(i, j)$. Put $p_{0}=0, p_{j+1}=p\left(p_{j}, 1\right)$ for $j \in \omega$ and denote $r_{j}^{\xi}=r_{\xi}\left(p_{j}, j\right), s_{j}^{\xi}=s_{\xi}\left(p_{j}, j\right)$. We can see that if $j \geq j_{\xi}$, then $\left[r_{j}^{\xi}, s_{j}^{\xi}\right) \subseteq\left[p_{j}, p_{j+1}\right), \bigcup_{r_{j}^{\xi} \leq k<s_{j}^{\xi}}\left[a_{k}^{\xi}, b_{k}^{\xi}\right) \subseteq\left[n_{p_{j}}, n_{p_{j+1}}\right)$ and $\sum_{r_{j}^{\xi} \leq k<s_{j}^{\theta}} \theta_{k}^{\xi} \sum_{a_{k}^{\xi} \leq n<b_{k}^{\xi}} \varrho_{n}^{\xi} \geq 1$.

For any $j \in \omega, \xi<\kappa$, we can code $\left\langle r_{j}^{\xi}, s_{j}^{\xi},\left\langle a_{k}^{\xi}, b_{k}^{\xi}, \theta_{k}^{\xi}\right\rangle_{p_{j} \leq k<p_{j+1}},\left\langle\varrho_{n}^{\xi}\right\rangle_{n_{p_{j}} \leq n<n_{p_{j+1}}}\right\rangle$ by $m_{j}^{\xi} \in \omega$. Since $\kappa<\operatorname{cov}(\mathbb{K})$, there exists a sequence $\left\{m_{j}\right\}_{j \in \omega}$ such that $\forall \xi<$ $\kappa \exists^{\infty} j m_{j}=m_{j}^{\xi}$. If $m_{j}=m_{j}^{\xi}$, let $r_{j}=r_{j}^{\xi}, s_{j}=s_{j}^{\xi}, a_{k}=a_{k}^{\xi}, b_{k}=b_{k}^{\xi}, \theta_{k}=\theta_{k}^{\xi}$ for $p_{j} \leq k<p_{j+1}$, and $\varrho_{n}=\varrho_{n}^{\xi}$ for $n_{p_{j}} \leq n<n_{p_{j+1}}$. Denote $M_{\xi}=\{j \in \omega: j \geq$ $\left.j_{\xi} \wedge m_{j}=m_{j}^{\xi}\right\}$ for $\xi<\kappa, M=\bigcup_{\xi<\kappa} M_{\xi}$ and $N=\omega \cap \bigcup_{j \in M}\left[r_{j}, s_{j}\right)$.

Now let us construct the perfect set $P$ by an induction on $k$. Put $\mathcal{T}_{0}=\left\{I_{0}\right\}$. Suppose that $\mathcal{T}_{k}$ is a family of intervals with pairwise disjoint interiors and that $|I| \geq \frac{1}{n_{k}}$ for every $I \in \mathcal{T}_{k}$.

If $k \in \omega \backslash N$, then $n_{k+1} \geq 2 n_{k}$ and by Lemma 2.3 for any $I \in \mathcal{T}_{k}$ there exists $J^{I} \subseteq I$ such that $\forall x \in J^{I}\left\|n_{k} x\right\| \leq \frac{n_{k}}{n_{k+1}}$ and $\left|J^{I}\right| \geq \frac{1}{n_{k+1}}$. Put $\mathcal{T}_{k+1}=\left\{J^{I}: I \in\right.$ $\left.\mathcal{T}_{k}\right\}$.

If $k \in N$, then $r_{j} \leq k<s_{j}$ for some $j \in M$. By Corollary 2.2 (1) there exists $J^{I} \subseteq I$ such that $\forall x \in J^{I}\left\|n_{k} x\right\| \leq \varepsilon_{j}$ and $\left|J^{I}\right| \geq \frac{\varepsilon_{j}}{n_{k}}$. Since $a_{k}<b_{k}, \theta_{k} \leq \frac{1}{14}$ and $\frac{\varepsilon_{j}}{n_{k}} \leq \frac{6 \theta_{k}}{a_{k}}$, by Lemma 2.9 there exists $\bar{J}^{I} \subseteq J^{I}$ such that

$$
\forall x \in \bar{J}^{I} \sum_{a_{k} \leq n<b_{k}} \varrho_{n}\|n x\| \geq \frac{\theta_{k}}{4} \sum_{a_{k} \leq n<b_{k}} \varrho_{n} \quad \text { and } \quad\left|\bar{J}^{I}\right| \geq \frac{2 \theta_{k}}{b_{k}} \geq \frac{2}{n_{k+1}} .
$$

Denote by $\bar{J}_{0}^{I}$ and $\bar{J}_{1}^{I}$ the left and the right half of $\bar{J}^{I}$ and put $\mathcal{T}_{k+1}=\left\{\bar{J}_{0}^{I}, \bar{J}_{1}^{I}: I \in\right.$ $\left.\mathcal{T}_{k}\right\}$.

Since $N$ is infinite, the set $P=\bigcap_{k \in \omega} \cup \mathcal{T}_{k}$ is a perfect subset of $I_{0}$. For all $x \in P$ we have $\lim _{k \rightarrow \infty}\left\|n_{k} x\right\|=0$, and hence $P \subseteq X$. Moreover, if $\xi<\kappa$ and $j \in M_{\xi}$ 
then

$$
\begin{aligned}
& \sum_{r_{j}^{\xi} \leq k<s_{j}^{\xi}} \sum_{a_{k}^{\xi} \leq n<b_{k}^{\xi}} \varrho_{n}^{\xi}\|n x\|=\sum_{r_{j} \leq k<s_{j}} \sum_{a_{k} \leq n<b_{k}} \varrho_{n}\|n x\| \\
& \geq \sum_{r_{j} \leq k<s_{j}} \frac{\theta_{k}}{4} \sum_{a_{k} \leq n<b_{k}} \varrho_{n}=\sum_{r_{j}^{\xi} \leq k<s_{j}^{\xi}} \frac{\theta_{k}}{4} \sum_{a_{k}^{\xi} \leq n<b_{k}^{\xi}} \varrho_{n}^{\xi} \geq \frac{1}{4},
\end{aligned}
$$

and hence $\sum_{n=0}^{\infty} \varrho_{n}^{\xi}\|n x\| \geq \sum_{j \in M_{\xi}} \sum_{r_{j}^{\xi} \leq k<s_{j}^{\xi}} \sum_{a_{k}^{\xi} \leq n<b_{k}^{\xi}} \varrho_{n}^{\xi}\|n x\|=\infty$, i.e. $x \notin Y_{\xi}$. We get $P \subseteq X \backslash \cup \mathcal{Y}$.

It is easy to see that for any increasing sequence $\left\{n_{k}\right\}_{k \in \omega}$ of positive integers there is a subsequence $\left\{n_{k_{j}}\right\}_{j \in \omega}$ such that $\lim _{j \rightarrow \infty} \frac{n_{k_{j}}}{n_{k_{j+1}}}=0$, or $\sum_{j=0}^{\infty} \frac{n_{k_{j}}}{n_{k_{j+1}}}<\infty$. Hence the family $\mathcal{F}$ of all sets $X$ satisfying the conditions of Theorem 2.5 (resp. $2.6,2.10)$ is a basis for the class $\mathcal{N}_{0}\left(\right.$ resp., $\left.\mathcal{B}_{0}, \mathcal{A}\right)$, i.e. every $\mathrm{N}_{0}$-set (resp. $\mathrm{B}_{0}$-set, A-set) is included in a set $X \in \mathcal{F}$. As immediate consequences we get the following:

Corollary 2.11. For any $N_{0}$-set $X$ there is an $N_{0}$-set $X^{\prime} \supseteq X$ such that for any family $\mathcal{Y}$ of $P D$-sets of size $|\mathcal{Y}|<\operatorname{add}(\mathbb{K})$ and for any interval $I_{0} \subseteq[0,1]$ with non-empty interior, the set $\left(X^{\prime} \backslash \bigcup \mathcal{Y}\right) \cap I_{0}$ has a perfect subset.

Corollary 2.12. For any $B_{0}$-set $X$ there is a $B_{0}$-set $X^{\prime} \supseteq X$ such that for any family $\mathcal{Y}$ of $P D$-sets of size $|\mathcal{Y}|<\operatorname{add}(\mathbb{K})$, the set $X^{\prime} \backslash \bigcup \mathcal{Y}$ has a perfect subset.

Corollary 2.13. For any $A$-set $X$ there is an $A$-set $X^{\prime} \supseteq X$ such that for any family $\mathcal{Y}$ of $N$-sets of size $|\mathcal{Y}|<\operatorname{add}(\mathbb{K})$ and for any interval $I_{0} \subseteq[0,1]$ with nonempty interior, the set $\left(X^{\prime} \backslash \bigcup \mathcal{Y}\right) \cap I_{0}$ has a perfect subset.

We do not know whether a similar result holds true in case of N-sets (resp. B-sets). The following question is open:

Question 2.14. Is it true that for any $N$-set $X$ there are sequences $\left\{\varrho_{k}\right\}_{k \in \omega}$ and $\left\{n_{k}\right\}_{k \in \omega}$ such that $\sum_{k=0}^{\infty} \varrho_{k}=\infty, \sum_{k=0}^{\infty} \varrho_{k} \frac{n_{k}}{n_{k+1}}<\infty$ and $\sum_{k=0}^{\infty} \varrho_{k}\left\|n_{k} x\right\|<\infty$ for all $x \in X$ ?

\section{REFERENCES}

1. J. Arbault, Sur l'ensemble de convergence absolue d'une série trigonométrique, Bull. Soc. Math. France 80 (1952), 253-317. MR 14:1080d

2. T. Bartoszyński, Combinatorial aspects of measure and category, Fund. Math. 127 (1987), 225-239. MR 88m:04001

3. N. K. Bary, A Treatise on Trigonometric Series, Macmillan, New York, 1964. MR 30:1347

4. L. Bukovský, N. N. Kholshchevnikova and M. Repický, Thin sets of harmonic analysis and infinite combinatorics, to appear in Real Anal. Exchange. CMP 95:17

5. E. K. van Douwen, The integers and topology, Handbook of Set-Theoretic Topology (K. Kunen and J. E. Vaughan eds.), North-Holland, Amsterdam, 1984, pp. 111-167. MR 87f:54008

6. P. Eliaš, Systemization of classes of thin sets related to the absolute convergence of trigonometric series (in Slovak), Master thesis, Univerzita P. J. Šafárika, Košice, 1993.

7. S. Kahane, Antistable classes of thin sets in harmonic analysis, Illinois J. Math. 37 (1993), 186-223. MR 94g:43005

8. J. Marcinkiewicz, Quelques théorèmes sur les séries et les fonctions, Bull. Sém. Math. Univ. Wilno 1 (1938), 19-24.

9. A. W. Miller, Some properties of measure and category, Trans. Amer. Math. Soc. 266 (1981), 93-114. MR 84e:03058a

10. R. Salem, The absolute convergence of trigonometric series, Duke Math. J. 8 (1941), 317-334. MR 2:360f 
11. J. E. Vaughan, Small uncountable cardinals and topology, Open Problems in Topology (J. van Mill and G. M. Reed eds.), North-Holland, Amsterdam, 1990, pp. 195-216. MR 92c:54001

12. T. Viola, Sull'insieme dei punti di convergenza delle serie trigonometriche generali, Ann. Scuola Norm. Sup. Pisa (2) 4 (1935), 155-162.

MatematickÝ Ústav SAV, Jesenná 5, 04154 Košice, Slovakia

E-mail address: elias@duro.upjs.sk 